\title{
Does a Drama-Inspired 'Mirroring' Exercise Enhance Mathematical Learning?
}

\author{
Eleni Smyrnis and Paul Ginns \\ Faculty of Education and Social Work, The University of Sydney, Australia
}

\begin{abstract}
Learning from complex instructional materials typically requires sustained attention, but many learners - both children and adults - may find their minds 'wandering' when learning. Drama educators have argued that 'mirroring' exercises, where students in pairs or groups mirror each other's movements, improve attention; but, to date, evidence for this claim is anecdotal. This exploratory study investigated the effects of a prelearning mirroring intervention on mathematical performance from the perspective of cognitive load theory. Twenty-six university students engaged in a mindful and embodied mirroring activity before learning a rapid mental mathematics strategy. Effects on learning were evaluated using two mathematical problem-solving tests completed after learning the maths strategy. Compared to the non-mirroring condition, students in the mirroring condition solved subsequent test problems more quickly than those in the control condition. Initial evidence thus suggests a mirroring intervention may enhance the quality of learning during mathematics.
\end{abstract}

Keywords: cognitive load theory, embodied cognition, attention, drama education, mirroring

Cognitive load theory (CLT; Sweller, 2011) is a contemporary educational psychology theory that applies findings from cognitive science to generate effective instructional designs for learning. The theory stipulates a working memory (WM) whose capacity and duration limitations can be circumvented by knowledge in schematic form in long-term memory. Baddeley's (1986) WM model has underpinned the human cognitive architecture used by CLT to generate novel instructional designs. Recently, Schweppe and Rummer (2014) have argued CLT might benefit from considering other WM models as a means of generating novel instructional designs. For example, Kane and Engle's (2002) WM model holds that WM capacity is a function of the ability to control attention, so that information is held in an active, easily retrievable state. Kane and Engle's WM model raises questions as to what instructional activities or designs might best enhance attention control; or, to put it another way, reduce mind wandering as a failure of attentional control. Mind wandering, defined by McVay and Kane (2010) as 'off-task thoughts during an ongoing task or activity' (p. 188), is one of the most ubiquitous of all mental activities (Killingsworth \& Gilbert, 2010),

Received 15 June 2016; Accepted 05 October 2016

Address for correspondence: Eleni Smyrnis, Faculty of Education and Social Work, University of Sydney NSW 2006, Australia. Email: esmy4411@uni.sydney.edu.au. 
and its impact in educational settings is just beginning to be understood (Smallwood, Fishman, \& Schooler, 2007; Wammes, Boucher, Seli, Cheyne, \& Smilek, 2016). Instructional designs to support attentiveness and/or reduce mind wandering thus stand in contrast to the traditional focus of CLT instructional designs on managing WM capacity limitations through a focus on intrinsic cognitive load (a function of the complexity of the learning task given the learner's knowledge base) and/or extraneous cognitive load (resulting from cognitive processes unrelated to learning).The current iteration of cognitive load theory incorporates insights from evolutionary theorising, holding that humans have evolved to attend to, process, and guide behavioural responses through biologically primary knowledge (Geary, 2008). Examples of biologically primary knowledge include facial recognition, physical gestures, and learning a native language. In contrast, a separate category of biologically secondary knowledge requires extensive, effortful engagement in learning, often through structured activity within educational institutions; examples include learning to read, write, and solve mathematical problems. Recent research informed by both embodied cognition perspectives and cognitive load theory has focused on both observed gestures (e.g., Wong et al., 2009) and self-generated gestures (e.g., Ginns, Hu, Byrne, \& Bobis, 2016) as forms of biologically primary knowledge that might support the construction of biologically secondary knowledge (see Paas \& Sweller, 2012). We propose that a specific drama education activity involving movement, mirroring, may likewise incorporate biologically primary behaviours and cognitive processes that can support learning by enhancing attention. In this practice, 'two actors face each other and mirror each other's movements' (Cohen, 2013, p. 203). One participant may begin as the leader and one as the follower, while maintaining eye contact throughout the process (Boal, 2002; Cohen, 2013). Cohen (2013) argues mirroring forces 'total situational concentration' in 'driving out all contextual awareness' (p. 203). According to these arguments, then, mirroring is an embodied drama activity that might fruitfully be incorporated into lessons requiring sustained attention.

Such effects on attention and learning can be predicted by several biologically primary attentional pathways. The first is 'a bias to orient to the human face and to automatically process key pieces of facial information' (Geary, 2008, p. 180; see also von Grünau \& Anston, 1995). Attention capture by gaze can be attributed to the social significance and survival value conveyed by eye contact (Baron-Cohen, 1995). The second potential attentional pathway mirroring may activate is through simple social presence. Ybarra et al. (2008) randomly assigned participants to conditions involving 10 minutes of social interaction, involving discussion of a social issue, 10 minutes of intellectual activities, or 10 minutes watching a video clip with social interactions. Participants in the social interaction condition outperformed the control participants on cognitive assessments of speed of processing and working memory, and performed at levels equivalent to the intellectual activities condition. Ybarra et al. explained these results by proposing that 'social interaction can "exercise" general cognitive processes (working memory, speed of processing, inhibition) in the service of social cognition (e.g., empathy, mentalizing, symbolic interaction)' (p. 255). Third, social interactions may often result in non-conscious mimicry of a range of nonverbal behaviours, including postures, mannerisms, and expressions (Chartrand \& Bargh, 1999). Vicaria and Dickens (in press) describe interpersonal coordination behaviours as a 'social glue' with evolutionary advantages, and their meta-analysis of the interpersonal coordination literature established reliable effects of such behaviours 
across a range of intrapersonal and interpersonal outcomes; this body of research did not, however, include any studies with an educational focus.

Anecdotal arguments made by drama educators thus suggest mirroring enhances attention through mimicking in a social context. The present study hypothesised that mirroring will temporarily enhance attention, in turn enhancing learning. Thus, a precursor mirroring activity may be an effective 'warm-up' activity prior to a mathematical lesson where sustained attention is required. We propose the following hypotheses:

1. Participants who begin a short mathematics lesson with a mirroring exercise will develop more effective problem-solving schemas than participants who perform the same movements without mirroring, as measured by subsequent test performance.

2. Participants who begin a short mathematics lesson with a mirroring exercise will develop more efficient problem-solving schemas than participants who perform the same movements without mirroring, as measured by subsequent time to solution on test questions.

\section{Method}

Participants

Twenty-six university students from The University of Sydney, the University of New South Wales, and the University of Technology Sydney (17 females, 9 males) participated in this study. All participants participated voluntarily and were aged between 18 and 24 years old $(M=20.80, S D=1.51)$. Participants were randomly and equally assigned to the mirroring (experimental) or non- mirroring (control) conditions. Students selected were novices in their ability to mentally compute and multiply two 2-digit numbers (i.e., without using any external resources such as pen and paper or a calculator).

\section{Materials and Procedure}

The project was approved by The University of Sydney Human Research Ethics Committee (protocol no. 2015/311). Participants were presented with a number of paperbased materials. These included: (1) demographic questions and pretest instructions, (2) a single page mathematical pretest, (3) four pages of acquisition material on a mental mathematics strategy for multiplication of two 2-digit numbers; (4) knowledge test instructions; and (5) a two-page problem-solving test.

Pretest on mathematics. A brief mathematical assessment (BMA-3) developed by Steiner and Ashcraft (2012) assessed participants' prior knowledge of a broad range of mathematical concepts. Participants had 6 minutes to complete 10 questions. This particular test was useful for measuring individuals' overall mathematical achievement as a potential confounding variable, given the present study's focus of mathematical learning.

Prelesson intervention. Two separate set of instructions were used by the experimenter to deliver the intervention: one for the mirroring group and one for the non-mirroring group. The instructions were taken from pedagogies described by Emunah (1994), 
Boal (2002), and Cohen (2013). Individually, each participant took part in either four mirroring or four non-mirroring exercises for 4 minutes.

The instructions for the mirroring (experimental) condition were as follows:

1. Mirroring clap: The leader held their hands together in front of them as if they were to clap. The follower was instructed to clap at the same time as the leader. Switching from the leader to the follower followed this.

2. Space counting: First, the experimenter began as the leader and the other as the mirror. The leader drew numbers using his/her index finger from 1 to 9 while the mirroring participant mirrored the leader with their index finger. After this was complete, they swapped roles.

3. Morning routine: From the previous activity, the experimenter leader improvised their morning routines (i.e., brushing their teeth, eating breakfast, washing their hair, waking up) while the mirroring participant mirrored the image. The experimenter encouraged participants to use their entire body.

4. Magnifying: The experimenter performed small incremental movement while the mirroring participant magnified that movement. An example could be a small clap by the leader causes the mirroring participant to clap more loudly.

The instructions for the non-mirroring (comparison) condition were identical to the above, except that all mirroring components were removed, and participants completed the movements by themselves.

Mental mathematics lesson. A one-page instruction sheet outlined that students should pay attention when reading through and learning a mental mathematics strategy — the multiplication of two 2-digit numbers - by studying four worked examples demonstrating the strategy. Worked examples were drawn from Julius' (1992, pp. 8384) text on rapid mental mathematics. Examples began with two of Julius' (1992) 'Elementary' questions (p. 83; e.g., $22 \times 14$ ), followed by two 'Brain Builder' questions (p. 84; e.g., $58 \times 85$ ), with each worked example presented on a single page. Students were given a total of 8 minutes to learn the strategy, 2 minutes for each worked example.

Knowledge tests. A one-page instruction sheet outlined the nature of the two tests. Both tests, drawn from Julius (1992), assessed fluency with the novel maths strategy. The first single page test comprised 10 Elementary questions (e.g., $12 \times 45)$ to which participants were allocated 5 minutes $(30$ seconds maximum per question to complete). The second single page test was comprised of 10 harder Brain Builder questions (e.g., $76 \times 54)$, to which participants were allocated 10 minutes ( 1 minute maximum per question). For all questions, one mark was allocated to a correct answer, and zero when the correct answer was not reached within the maximum time allowable.

\section{Results}

Descriptive statistics (means and standard deviations) for all variates under analysis are given in Table 1.

The Anderson-Darling test of normality was used to evaluate the assumption of normally distributed scores in each condition, and followed Keselman, Othman, and 
TABLE 1

Descriptive Statistics for Variates Under Analysis

\begin{tabular}{lcc}
\hline & Non-mirroring & \multicolumn{1}{c}{ Mirroring } \\
\hline Brief Math Assessment Pretest (/10) & $7.69(1.25)$ & $8.00(1.08)$ \\
Test 1 - Elementary & & \\
Time to correct solution (sec.) & $212.46(30.72)$ & $182.38(38.96)$ \\
Number of correct solutions (/10) & $9.00(.91)$ & $9.23(1.17)$ \\
Test 2 - Brain Builders & & \\
Time to correct solution (sec.) & $303.08(65.87)$ & $237.85(73.95)$ \\
Number of correct solutions (/10) & $9.69(.48)$ & $9.69(0.85)$ \\
\hline
\end{tabular}

Wilcox's (2013) recommendation of a type 1 error rate for this test of .20. Using this standard, data were found to be non-normally distributed in one or both conditions for several of the variables under analysis. Levene's test, which is frequently used to test the assumption of homogeneity of variances across experimental conditions, is also dependent on the assumption of normality. The recently developed nonparametric Levene test (Nordstokke, Zumbo, Cairns, \& Saklofske, 2011), based on a rank transformation of all scores followed by Levene's test on these scores, provides a suitable alternative under violations of the assumption of normality.

Since for some variates data were non-normally distributed but the non-parametric Levene test was not statistically significant, the Mann-Whitney $U$ test using an exact $p$ value (Mehta \& Patel, 2012) was used to analyse these data. Additional to these analyses, standardised mean difference effect size, Cohen's $d$, with its $95 \%$ confidence interval is also reported (for Mann-Whitney tests, effect sizes and confidence intervals are based on methods developed by Newcombe, 2006a, 2006b). The present study adopted Hattie's (2009) benchmarks for effect sizes in educational studies suggesting small $d=.20$, medium $d=.40$, and large $d=.60$ and above. Analyses controlled the Type 1 error rate at 0.05 .

\section{Were the Groups Equivalent in Prior Knowledge?}

A preliminary analysis investigated whether the mirroring and non-mirroring conditions were equivalent in general mathematical knowledge by analysing participants' Brief Mathematics Assessment-3 scores. The Anderson-Darling test of normality verified that the distribution of scores in each condition was not normally distributed with the mirroring condition $(p<.001)$ and non-mirroring condition $(p=.101)$. A non-parametric Levene test verified the equality of variances across the experimental conditions $(F=3.88, p=.061)$. A Mann-Whitney test determined that there was no significant difference between the mirroring group (mean rank $=14.62$ ) and the non-mirroring group (mean rank $=12.38$ ) on the Brief Math Assessment scores; $U=$ $70.00, p=.444, d=.31$ [95\% CI -.47, 1.05]. Thus, random assignment of participants to conditions was effective in distributing mathematical ability across conditions.

Does a Mirroring Intervention Improve Efficiency of Mathematics and Mathematical Learning Outcomes?

The major variables under analysis were number of correct solutions to problems (hypothesis 1 - effectiveness of learning) and time to correct solution across the 
two tests (hypothesis 2 - efficiency of learning). For Elementary test questions, a ceiling effect was obtained on number of questions correctly answered (mirroring condition mean score $=92.3 \%$ correct; non-mirroring condition mean score $=90 \%$ ), precluding analysis of this variate. For time to solution of Elementary test questions, the Anderson-Darling test of normality showed that both the mirroring $(p=.249)$ and non-mirroring data $(p=.211)$ were normally distributed. Subsequently, a non-parametric Levene test verified the equality of variances in the samples, which were not statistically significant $(F=.212, p=.649)$. An independent groups $t$ test established that participants in the mirroring condition solved the Elementary test questions more quickly $(M=182.38$ seconds, $S D=38.96)$ than the non-mirroring condition $(M=212.46, S D 30.72), t(24)=-2.19, p=.039, d=-.86,95 \%$ CI $[-1.66$, -0.05]. Thus, hypothesis 1 of the study was not supported in the number of questions correctly answered. However, participants in the mirroring group were more efficient in answering these questions by the lower amount of time taken to answer questions, compared to the non-mirroring group, supporting hypothesis 2 .

As with the Elementary test questions, a ceiling effect was obtained on number of Brain Builder questions correctly answered (mirroring condition mean score $=96.9 \%$ correct; non-mirroring condition mean score $=96.9 \%$ ), precluding analysis of this variate. For time to solution of Brain Builder test questions, the Anderson-Darling test of normality showed that the mirroring condition was not normally distributed ( $p=.117)$, whereas the non-mirroring group was normally distributed ( $p=.976)$. As these results varied across conditions, a conservative approach is to infer that the assumption of normality was not met. Subsequently, the non-parametric Levene test showed the equality of variance in the samples was not statistically significant $(F=.358, p=.555)$. The Mann-Whitney non-parametric test found the mirroring condition solved the more complex 'Brain Builder' test questions more quickly (mean rank $=9.88$ ) than the non-mirroring condition (mean rank $=17.12), U=37.50$, $p=.015, d=-1.08$ [95\% CI -1.83, -.19]. Thus, hypothesis 1 of the study was also not supported in the number of questions correctly answered in this harder test. However, participants in the mirroring group were also considerably more efficient in answering the harder questions, supporting hypothesis 2.

\section{Discussion}

The results of this exploratory experiment provide initial evidence for a prelearning mirroring exercise to enhance learning. We first hypothesised that participants in the mirroring group would outperform participants in the non-mirroring group on a subsequent test of problem solving. The results in the present study were limited by the ceiling effects obtained for both the Elementary and Brain Builders tests. The second hypothesis predicted participants in the mirroring group would solve test problems more quickly than those in the non-mirroring group. Results for time to solution in both tests indicated participants in the mirroring group were more efficient in answering questions in both of the above tests, measured by the shorter times taken to answer questions. The effects of mirroring on problem-solving efficiency were large for both tests (Elementary: $d=-.86$; Brain Builders: $d=-1.08$ ) using Hattie's (2009) suggested benchmarks. The present study thus provides initial evidence for the potential of the mirroring activity to support university students' mathematical learning. 
Ceiling effects found across both Elementary and Brain Builder tests were limitations in this study. The results reported above speak to the significance in instructional design experiments of suitable alignment between the prior knowledge levels of participants and the complexity of the materials and test questions (Cooper \& Sweller, 1987). The high level of prior knowledge and test performance achieved here informs two approaches for improving the design of the next investigation of the mirroring effect. First, recruiting a wider range of students in future research (e.g., non-university students) would likely reduce the likelihood of a ceiling effect on test scores. Second, increasing the level of difficulty of problems, from the multiplication of two 2-digit numbers to the multiplication of two 3 -digit numbers, should also avoid ceiling effects.

This pilot study forms the beginning of a planned program of research. Beyond establishing a mirroring effect on test scores through the changes described above, we also plan to investigate the 'dosage' of mirroring time needed to give an effective learning boost to participants. Future studies will also investigate process variables as the basis for theorising about reducing mind-wandering. Seli, Risko, and Smilek (2016) used randomly spaced probes during an experimental session to measure whether participants were 'on-task' or 'mind wandering'; such instrumentation provides a potential model for measuring mind-wandering in future studies of the mirroring effect.

In summary, this study found that a mirroring intervention prior to mathematical learning improved a student's efficiency in subsequent mathematical problem-solving. From a theoretical perspective, the study provides an initial attempt to broaden the range of working memory architectures considered by CLT (cf. Schweppe \& Rummer, 2014), but further research will be needed to provide evidence that the mirroring effect occurs through attentional control mechanisms.

\section{Financial Support}

This research received no specific grant from any funding agency, commercial, or not-for-profit sectors.

\section{Conflicts of Interest}

This research received no specific grant from any funding agency, commercial, or not-for-profit sectors.

\section{Ethical Standards}

The authors assert that all procedures contributing to this work comply with the ethical standards and legal requirements of the relevant national and institutional committees on human experimentation and with the Helsinki Declaration of 1975, as revised in 2008 .

\section{References}

Baddeley, A. (1986). Working memory. Oxford University Press.

Baron-Cohen, S. (1995). The eye direction detector (EDD) and the shared attention mechanism (SAM): Two cases for evolutionary psychology. In C. Moore \& P.J. Dunham, (Eds.), Joint attention: Its origins and role in development (pp. 41-59). Hillsdale, NJ: Lawrence Erlbaum.

Boal, A. (2002). Games for actors and non-actors. New York, NY: Psychology Press. 
Chartrand, T.L., \& Bargh, J.A. (1999). The chameleon effect: The perception-behavior link and social interaction. Journal of Personality and Social Psychology, 76, 893-910.

Cohen, R. (2013). Acting power: The 21st century edition. London, England: Taylor \& Francis.

Cooper, G., \& Sweller, J. (1987). Effects of schema acquisition and rule automation on mathematical problem-solving transfer. Journal of Educational Psychology, 79, 347-362.

Emunah, R. (1994). Acting for real: Drama therapy process, technique, and performance. New York, NY: Brunner/Mazel Publishers.

Geary, D.C. (2008). An evolutionarily informed education science. Educational Psychologist, 43, 179-195.

Ginns, P., Hu, F.-T., Byrne, E., \& Bobis, J. (2016). Learning by tracing worked examples. Applied Cognitive Psychology, 30, 160-169.

Hattie, J. (2009). Visible learning: A synthesis of over 800 meta-analyses relating to achievement. New York, NY: Routledge.

Julius, E. (1992). Rapid math. New York, NY: Wiley.

Kane, M.J., \& Engle, R.W. (2002). The role of prefrontal cortex in working-memory capacity, executive attention, and general fluid intelligence: An individual-differences perspective. Psychonomic Bulletin \& Review, 9, 637-671.

Keselman, H.J., Othman, A.R., \& Wilcox, R.R. (2013). Preliminary testing for normality: Is this a good practice? Journal of Modern Applied Statistical Methods, 12., Article 2. Retrieved from http://digitalcommons.wayne.edu/jmasm/vol12/iss2/2

Killingsworth, M.A., \& Gilbert, D.T. (2010). A wandering mind is an unhappy mind. Science, 330, 932-932.

McVay, J.C., \& Kane, M.J. (2010). Does mind wandering reflect executive function or executive failure? Comment on Smallwood and Schooler (2006) and Watkins (2008). Psychological Bulletin, 136, 188207.

Mehta, C.R., \& Patel, N.R. (2012). IBM SPSS exact tests. Retrieved from ftp://public.dhe.ibm.com/ software/analytics/spss/documentation/statistics/21.0/en/client/Manuals/IBM_SPSS_Exact_Tests.pdf

Newcombe, R. (2006a). Confidence intervals for an effect size measure based on the Mann-Whitney statistic. Part 1: General issues and tail-area-based methods. Statistics in Medicine, 25, 543-557.

Newcombe, R. (2006b). Confidence intervals for an effect size measure based on the Mann-Whitney statistic. Part 2: Asymptotic methods and evaluation. Statistics in Medicine, 25, 559-573.

Nordstokke, D.W., Zumbo, B.D., Cairns, S.L., \& Saklofske, D.H. (2011). The operating characteristics of the nonparametric Levene test for equal variances with assessment and evaluation data. Practical Assessment, Research \& Evaluation, 16. Retrieved from: http://pareonline.net/getvn.asp? $\mathrm{v}=16 \& \mathrm{n}=5$.

Paas, F., \& Sweller, J. (2012). An evolutionary upgrade of cognitive load theory: Using the human motor system and collaboration to support the learning of complex cognitive tasks. Educational Psychology Review, 24, 27-45.

Seli, P., Risko, E.F., \& Smilek, D. (2016). On the necessity of distinguishing between unintentional and intentional mind wandering. Psychological Science, 27, 685-691.

Steiner, E.T., \& Ashcraft, M.H. (2012). Three brief assessments of math achievement. Behavior Research Methods, 44, 1101-1107.

Sweller, J. (2011). Cognitive load theory. In J. Mestre \& B.H. Ross (Eds.), The psychology of learning and motivation; Cognition in education (vol. 55, pp. 77-76). Oxford: Academic Press.

Schweppe, J., \& Rummer, R. (2014). Attention, working memory, and long-term memory in multimedia learning: An integrated perspective based on process models of working memory. Educational Psychology Review, 26, 285-306.

Smallwood, J., Fishman, D.J., \& Schooler, J.W. (2007). Counting the cost of an absent mind: Mind wandering as an underrecognized influence on educational performance. Psychonomic Bulletin \& Review, 14, 230-236.

Vicaria, I.M., \& Dickens, L. (in press). Meta-analyses of the intra-and interpersonal outcomes of interpersonal coordination. Journal of Nonverbal Behavior.

von Grünau, M., \& Anston, C. (1995). The detection of gaze direction: A stare-in-the-crowd effect. Perception, 24, 1297-1313. 
Wammes, J.D., Boucher, P.O., Seli, P., Cheyne, J.A., \& Smilek, D. (2016). Mind wandering during lectures I: Changes in rates across an entire semester. Scholarship of Teaching and Learning in Psychology, 2, 33-48.

Wong, A., Marcus, N., Ayres, P., Smith, L., Cooper, G.A., Paas, F., \& Sweller, J. (2009). Instructional animations can be superior to statics when learning human motor skills. Computers in Human Behavior, 25, 339-347.

Ybarra, O., Burnstein, E., Winkielman, P., Keller, M.C., Manis, M., Chan, E., \& Rodriguez, J. (2008). Mental exercising through simple socializing: Social interaction promotes general cognitive functioning. Personality and Social Psychology Bulletin, 34, 248-259. 